\title{
Special issue on cognitive-inspired computing and applications
}

\author{
Jun $\mathrm{Ye}^{1} \cdot$ Sulin Pang ${ }^{2}$
}

Received: 6 September 2021 / Accepted: 6 September 2021 / Published online: 16 September 2021

(C) The Author(s), under exclusive licence to Springer-Verlag London Ltd., part of Springer Nature 2021

Cognition is emerging as a new and promising methodology with the development of cognitive-inspired computing, cognitive-inspired interaction and systems, which has the potential to enable a large class of applications and has emerged with a great potential to change our life. However, recent advances on artificial intelligence (AI), fog computing, big data, and cognitive computational theory show that multidisciplinary cognitive-inspired computing still struggle with fundamental, long-standing problems, such as computational models and decision-making mechanisms based on the neurobiological processes of the brain, cognitive sciences, and psychology. How to enhance human cognitive performance with machine learning, common sense, natural language processing, etc. are worth exploring. The following articles in this special issue introduce a variety of cognitive-inspired computing and applications.

$\mathrm{Yu}$ et al. [1] propose a Model-based Collaborate Filtering Algorithm Based on Stacked AutoEncoder (MCFSAE) to overcome the sparsity problem. Zhang and Hao [2] start from the analysis of the disaster mechanism and attribute characteristics of mountain floods and proposes an RBF neural network integration based on rough set flood damage loss assessment model of, and the flood loss assessment model based on neural network integration was applied in the flood loss prediction work of Village A, making the flood loss rate suitable for the use of the flood loss assessment model. Guan and Wang [3] construct a gray fuzzy prediction model based on neural network, a gray extreme learning machine prediction model, and a gray fuzzy extreme learning machine prediction combination model based on neural network. Peng [4] constructs an intelligent English network learning system based on the

\section{Jun Ye}

yejun@suse.edu.cn

1 Hainan University, No 58, Renmin Avenue, Haikou 570228, Hainan province, China

2 Jinan University, 601 Huangpu West Avenue, Tianhe, Guangzhou, Guangdong, China improved algorithm. Wu et al. [5] regard the discovery and scheduling problem of replaceable services replacement as the discovery and scheduling component, and develop an approach based on replaceable service form the app vendor's perspective for solving the none replaceable services environment. Yang and Liao [6] introduce the time dimension to describe the evolutionary characteristics of enterprise risk events, such as dynamics, suddenness and timeliness. Wang and $\mathrm{Mu}$ [7] propose a method of dividing the error by the proportion of each link's contribution to the error, and the idea that the error of the hidden layer node is the sum of the errors on each link during the forward propagation process. Jiang and Zhao [8] combine GPS occultation technology to improve the algorithm and construct the boundary layer height simulation model based on the actual situation and analyze its functional structure one by one. Zhu et al. [9] use the method of support vector neural network to predict and analyze China's foreign trade export and uses principal component analysis and regression analysis to analyze the contribution rate of different influencing factors to foreign trade export. Hua et al. [10] design a vision system based on a high-resolution color camera and TOF depth camera.

Yang et al. [11] take the skyline probability calculation and the actual efficiency of the unknown object index structure in the skyline query processing technology on the uncertain data stream as the research object. Zhang [12] aims to study the prediction and prediction of rockburst risk based on particle swarm algorithm and neural network. Jiang et al. [13] take the Shanghai Composite Index and three listed stocks in the stock market as examples, collects the closing data of the Shanghai Composite Index and the stock market prices of three listed companies as sample data, uses the BP neural network prediction model and the optimized particle swarm optimization-neural networks (PSO-BP) neural network model predicts the future trends of the Shanghai Composite Index and the three stocks, respectively. Zhou et al. [14] adopt the fuzzy information entropy-based fuzzy rough set to conduct redundant APs reduction. Guo et al. [15] adopted to reduce the dimensionality of the data. Yao et al. [16] complete the control 
operation of the embedded machine vision system on the video processing in the pipeline. Zhu et al. [17] focus on the two-dimensional facial expression animation technology under DNN. Ruan et al. [18] propose an innovative method to improve the attribute weighting approaches for naïve Bayes text classifiers using the improved distance correlation coefficient. Huang et al. [19] design a new segment attention mechanism based on Convolutional Neural Network, which enables extracting local semantic features through word embedding. Zhang and Li [20] compare the prediction performance of neural network prediction algorithm (NN), genetic algorithm, and support vector machine-based machine learning algorithm (SVM) and uses statistical analysis to perform data analysis and draw corresponding curves.

\section{References}

1. Yu M, Quan T, Peng Q et al (2021) A model-based collaborate filtering algorithm based on stacked AutoEncoder. Neural Comput \& Applic. https://doi.org/10.1007/s00521-021-05933-8

2. Zhang Y, Hao Y (2021) Loss prediction of mountain flood disaster in villages and towns based on rough set RBF neural network. Neural Comput \& Applic. https://doi.org/10.1007/s00521021-05902-1

3. Guan S, Wang X (2021) Optimization analysis of football match prediction model based on neural network. Neural Comput \& Applic. https://doi.org/10.1007/s00521-021-05930-x

4. Peng N (2021) Research on the effectiveness of English online learning based on neural network. Neural Comput \& Applic. https://doi.org/10.1007/s00521-021-05855-5

5. Wu Z, Yan Z, Huang D et al (2021) An approach for discovery and scheduling replaceable service on edge environment. Neural Comput \& Applic. https://doi.org/10.1007/s00521-021-05862-6

6. Yang B, Liao Ym (2021) Research on enterprise risk knowledge graph based on multi-source data fusion. Neural Comput \& Applic. https://doi.org/10.1007/s00521-021-05985-w

7. Shuo W, Ming M (2021) Exploring online intelligent teaching method with machine learning and SVM algorithm. Neural Comput \& Applic. https://doi.org/10.1007/s00521-021-05846-6

8. Jiang R, Zhao K (2021) Using machine learning method on calculation of boundary layer height. Neural Comput \& Applic. https://doi.org/10.1007/s00521-021-05865-3
9. Han Z, Zhu Z, Zhao S et al (2021) Research on nonlinear forecast and influencing factors of foreign trade export based on support vector neural network. Neural Comput \& Applic. https://doi.org/ 10.1007/s00521-021-05900-3

10. Ding Y, Hua L, Li S (2021) Research on computer vision enhancement in intelligent robot based on machine learning and deep learning. Neural Comput \& Applic. https://doi.org/10.1007/ s00521-021-05898-8

11. Zeng Y, Yang Z, Zhang W et al (2021) Application of processing technology based on skyline query in computer network. Neural Comput \& Applic. https://doi.org/10.1007/s00521-021-05931-w

12. Zhang M (2021) Prediction of rockburst hazard based on particle swarm algorithm and neural network. Neural Comput \& Applic. https://doi.org/10.1007/s00521-021-06057-9

13. Jiang Y (2021) Prediction model of the impact of innovation and entrepreneurship on China's digital economy based on neural network integration systems. Neural Comput \& Applic. https:// doi.org/10.1007/s00521-021-05899-7

14. Nie W, Liu Z, Zhou M et al (2021) Joint access point fuzzy rough set reduction and multisource information fusion for indoor Wi-Fi positioning. Neural Comput \& Applic. https://doi.org/10.1007/ s00521-021-05934-7

15. Zeng Y, Guo Y, Li J (2021) Recognition and extraction of highresolution satellite remote sensing image buildings based on deep learning. Neural Comput \& Applic. https://doi.org/10.1007/ s00521-021-06027-1

16. Song Z, Yao J, Hao H (2021) Design and implementation of video processing controller for pipeline robot based on embedded machine vision. Neural Comput \& Applic. https://doi.org/10. 1007/s00521-021-06022-6

17. Xu P, Zhu Y, Cai S (2021) Innovative research on the visual performance of image two-dimensional animation film based on deep neural network. Neural Comput \& Applic. https://doi.org/ 10.1007/s00521-021-06140-1

18. Ruan S, Chen B, Song K et al (2021) Weighted naïve Bayes text classification algorithm based on improved distance correlation coefficient. Neural Comput \& Applic. https://doi.org/10.1007/ s00521-021-05989-6

19. Bai T, Guan H, Wang S et al (2021) Traditional Chinese medicine entity relation extraction based on $\mathrm{CNN}$ with segment attention. Neural Comput \& Applic. https://doi.org/10.1007/ s00521-021-05897-9

20. Zhang L, Li N (2021) Material analysis and big data monitoring of sports training equipment based on machine learning algorithm. Neural Comput \& Applic. https://doi.org/10.1007/s00521021-05852-8

Publisher's Note Springer Nature remains neutral with regard to jurisdictional claims in published maps and institutional affiliations. 